\title{
A ROCKET OBSERVATION OF COSMIC X-RAYS IN THE ENERGY RANGE BETWEEN 0.15 AND $20 \mathrm{keV}$
}

\author{
S. HAYAKAWA, T. KATO, F. MAKINO, H. OGAWA, \\ Y. TANAKA, and K. YAMASHITA \\ Dept. of Physics, Nagoya University, Nagoya, Japan \\ and \\ M. MATSUOKA, S. MIYAMOTO, M. ODA and Y. OGAWARA \\ Institute of Space and Aeronautical Science, University of Tokyo, Tokyo, Japan
}

\begin{abstract}
Cosmic X-rays were observed with three sets of proportional counters covering the energy range between 0.15 and $20 \mathrm{keV}$. The detector born on a spinning rocket scanned a celestial region in which the galactic latitude $b^{\mathrm{II}}$ changed from $30^{\circ}$ to $-55^{\circ}$ across the galactic plane in the CygnusCassiopeia region. The spectrum of Cyg XR-2 thus obtained is represented by a thermal bremsstrahlung of temperature $3.4 \mathrm{keV}$ modified by the interstellar absorption for the hydrogen column density of $3 \times 10^{21} \mathrm{~cm}^{-2}$. The diffuse component showed an interstellar absorption effect, which was however found much weaker than one would expect if the diffuse component were due entirely to be of extragalactic origin. The spectrum obtained in the highest latitude region is represented approximately by a power law $E^{-1.8}$ but shows a possible trough at about $1 \mathrm{keV}$.
\end{abstract}

\section{Introduction}

Observation of cosmic X-rays at low energies is known to be important in understanding the mechanism of X-ray emission and investigating properties of interstellar matter through the absorption of X-rays. For this purpose detectors sensitive to soft $\mathrm{X}$-rays have to be used. This has been achieved by means of proportional counters with thin polypropylene windows.

In order to guarantee the reliability of experimental results, we used three kinds of counters whose sensitive regions partially overlap with each other. This also facilitated to obtain the energy spectrum in a wide energy range from $150 \mathrm{eV}$ to $20 \mathrm{keV}$.

The X-ray detector was launched on board a Japanese sounding rocket (K-10-4) at 19:00 (JST) 14 Jan. 1969 from Kagoshima Space Center $\left(31^{\circ} \mathrm{N}, 131^{\circ} \mathrm{E}\right)$, Institute of Space and Aeronautical Science, University of Tokyo. The rocket reached a maximum altitude of $226 \mathrm{~km}$. The detector scanned a sky region nearly parallel to the galactic longitude, so that the effect of interstellar absorption could be obtained. The scanning path crossed the galactic plane in Cygnus-Cassiopeia region, so that Cyg XR-2 was clearly resolved. An X-ray source which probably coincided with Cas $\mathrm{A}$ was also resolved with a reasonable confidence level, although its separation from near-by sources and possible $\mathrm{X}$-ray emission from the galactic disk was not yet unambiguous at the present stage of data analysis.

In the present paper, therefore, we restrict ourselves to results on the diffuse component and Cyg XR-2. 


\section{Instrumentation}

The detector consisted of seven proportional counters, of which three counters had 120 micron Be windows filled with $250 \mathrm{~mm} \mathrm{Hg}, \mathrm{Xe}+10 \% \mathrm{CH}_{4}$, the other four had $4 \mu$ thick polypropylene windows with $200 \AA$ aluminium coating. Two of them were filled with $\mathrm{Ar}+10 \% \mathrm{CH}_{4}$, and the other two with $\mathrm{He}+10 \% \mathrm{CH}_{4}$ of $1 \mathrm{~atm}$, respectively. The effective area of each counter was $50 \mathrm{~cm}^{2}$. Be counters covered the energy range 2-20 keV, whereas the polypropylene counters were also sensitive to soft X-rays of energies above $150 \mathrm{eV}$. The detection efficiency vs. energy is shown in Figure 1.

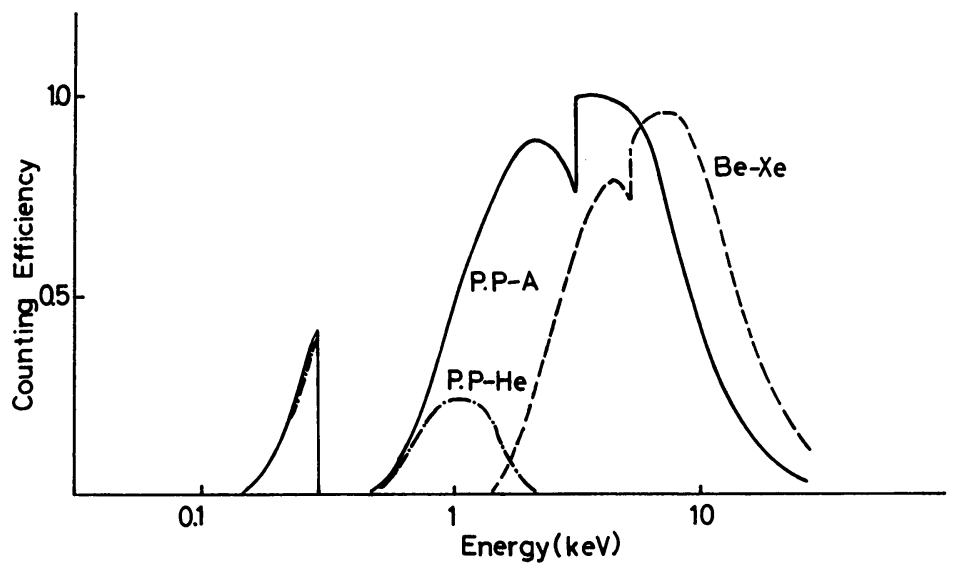

Fig. 1. Efficiencies of the counters calculated for X-rays of normal incidence.

The counter axis was inclined by $76^{\circ}$ with respect to the rocket axis and the field of view was defined by two sets of slats collimators (A and B), FWHM $4^{\circ} \times 15^{\circ}$. The collimator $\mathrm{A}$ was approximately parallel to the galactic plane in the Cygnus-Cassiopeia region and was placed in front of three Be counters and a pair of polypropylene counters, one Ar-filled and the other He-filled. The collimator B for another pair of Ar- and He-filled polypropylene counters was tilted by $70^{\circ}$ with respect to the collimator A.

A door-type shutter that periodically opened and closed the field of view was incorporated in order to measure the environmental background counts separately. Inflight calibration was provided throughout the flight with ${ }^{55} \mathrm{Fe}$ sources mounted on the shutter. A weak C-K X-ray source served to monitor the He-filled polypropylene counter that is insensitive to $\mathrm{K} \mathrm{X}$-rays from ${ }^{55} \mathrm{Fe}$.

Because of the high permeability of the polypropylene film for air, polypropylene counters were continuously flushed from an outside reservoir till the time of launch and were later closed by solenoid valves.

Pulse height of each count was telemetered to the ground. Inflight calibration data verified perfect functioning of the payload. 


\section{Performance of Experiment}

X-ray counts were obtained during about 200 sec. According to the roll of the rocket at a rate of $4.2 \mathrm{c} / \mathrm{sec}$ with a small yaw angle, the counters scanned a fixed band on the celestial sphere, including Cyg XR-2 and Cas A, as shown in Figure 2. The results of added counts for every spin as a function of roll angle are shown for individual sets of counters and for given energy ranges in Figure 3. The background counting rates

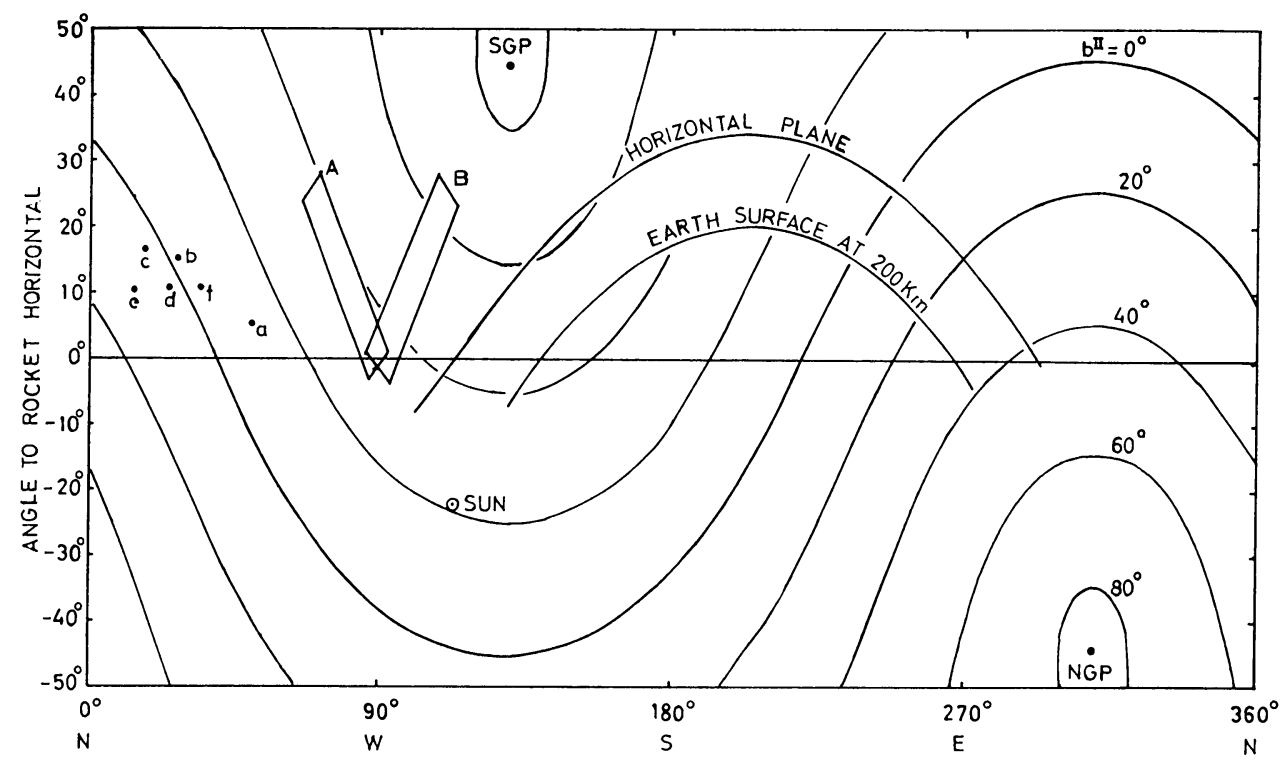

Fig. 2. The scanned band on the celestial sphere in the rocket frame of reference. The rocket axis pointed $\alpha=3^{\mathrm{h}} 19^{\mathrm{m}}$ and $\delta=0.5^{\circ}$, whereby a small yaw motion is neglected. The fields of view of collimators $\mathrm{A}$ and $\mathrm{B}$ are indicated. Curves represent galactic latitudes $b^{\mathrm{II}}$ and the horizons at the ground surface and at an altitude of $200 \mathrm{~km}$. The positions of the galactic north and south poles (N.G.P. and S.G.P., respectively), the sun and several known X-ray sources are indicated;

(a) Cyg XR-2; (b) Cas A; (c) Cep XR-1; (d) Cep XR-2; (e) Cep XR-3; (f) Lac XR-1.

as deduced from the closed-shutter period are indicated by dotted lines. They agree with the counting rates when looking at the earth in the energy range above $1 \mathrm{keV}$, so that the earth can also be used as a shutter. As energy decreases, however, the difference between the counting rates when looking at the earth and when the shutter was closed increases. There is a significant difference in the lowest energy region.

In this energy region a strong peak was found in the direction of the western horizon and a weak peak near the eastern horizon. Since the sun was located by $35^{\circ}$ below the counter axis when the axis passed by the direction indicated by an arrow in Figure 3, the western peak may be due either to scattered solar X-rays or to twilight glow. The counter was slightly sensitive to ultraviolet radiation of wavelengths below $3500 \AA$. The counters were subject to atmospheric background also in other directions. 


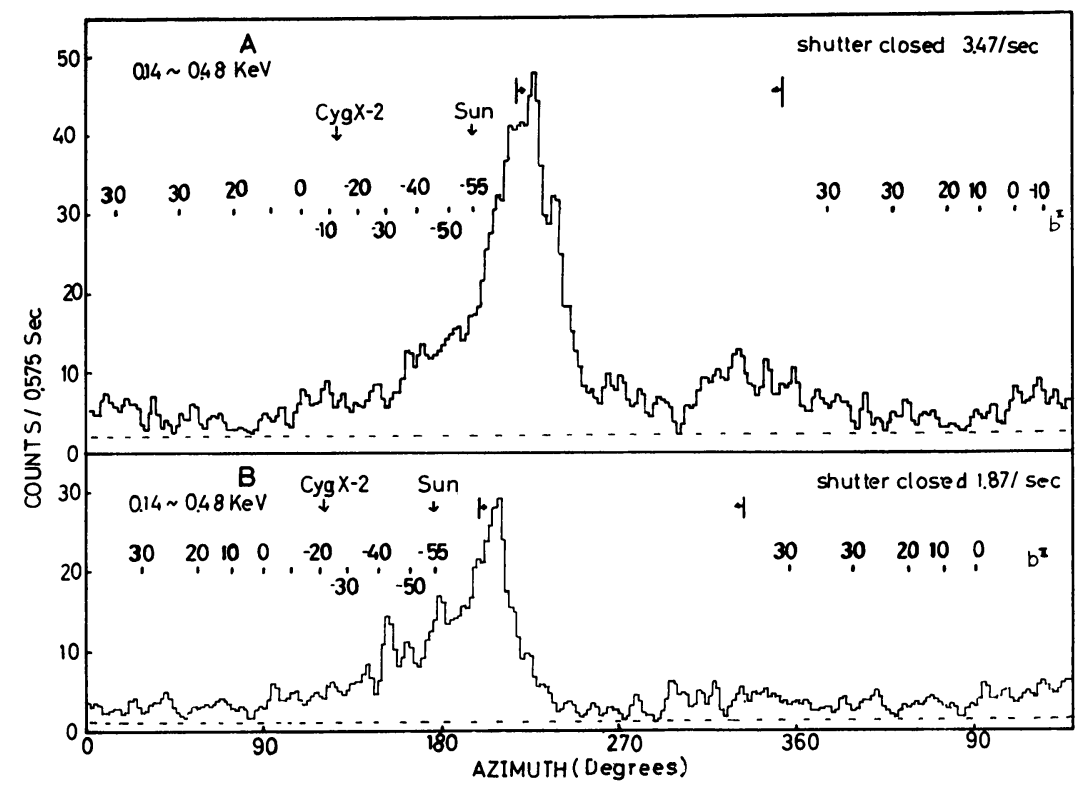

Fig. 3a.

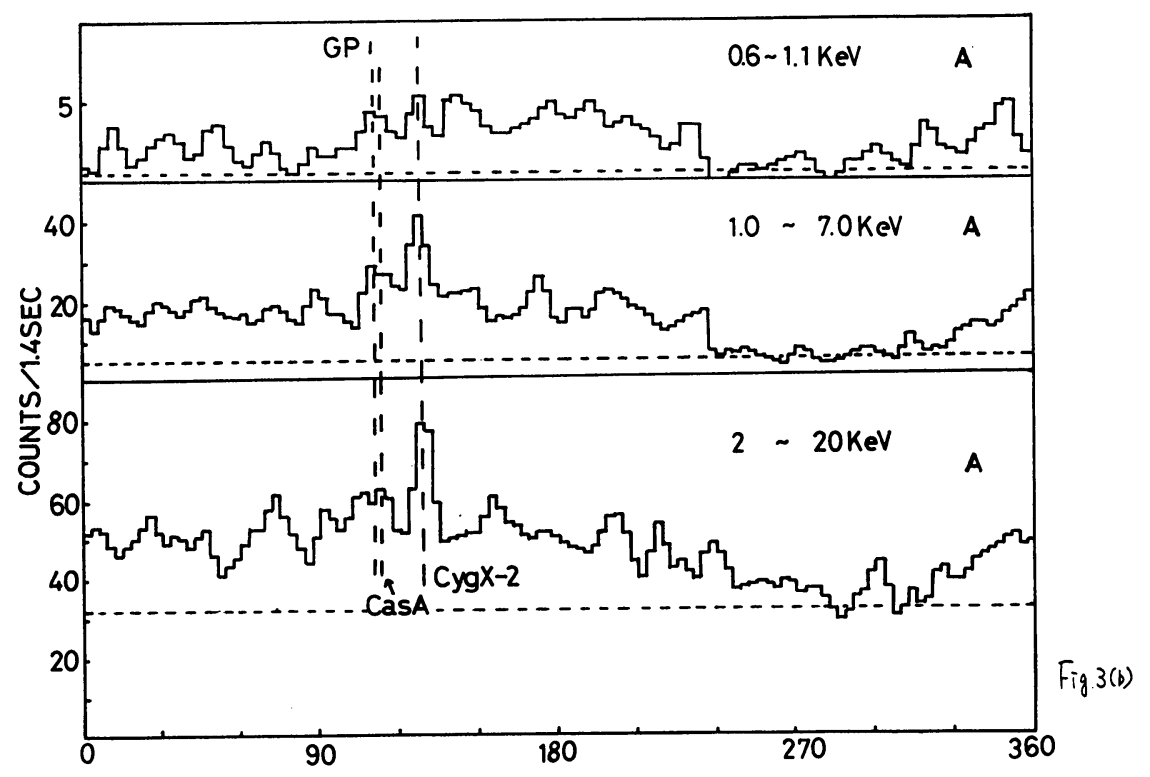

Fig. $3 b$. 


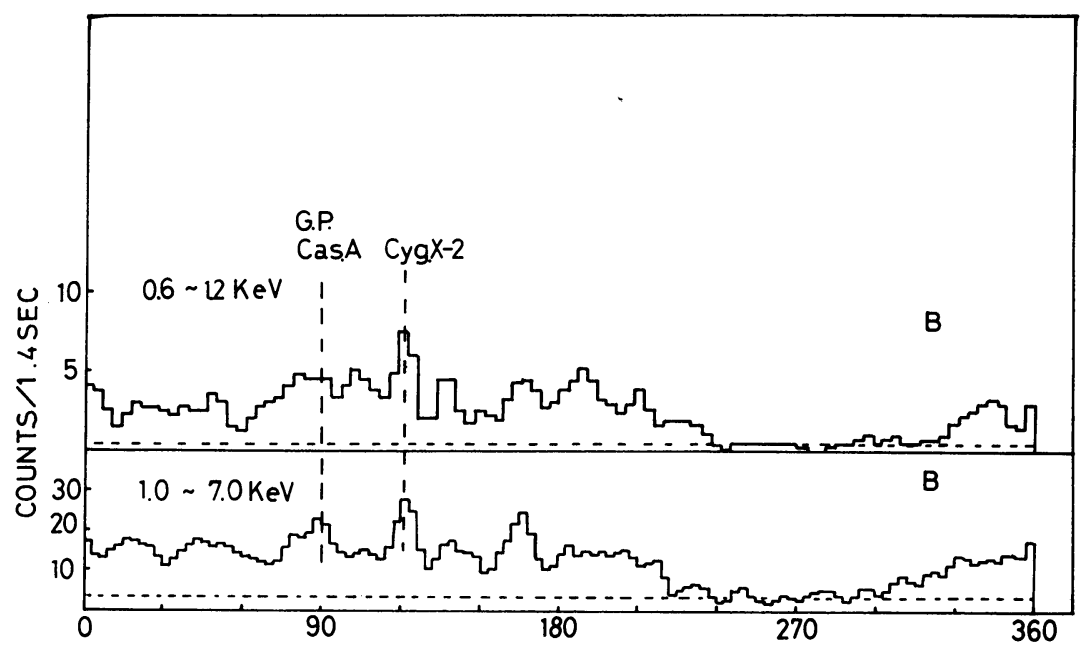

Fig. 3c.

Fig. 3. Counting rates vs. azimuth angle in the rocket frame of reference. - (a) Energy range 0.14-0.48 keV, collimators A and B, counts of He- and Ar-filled counters being added. - (b) Energy ranges $0.6-1.1 \mathrm{keV}, 1.0-7.0 \mathrm{keV}$ and 2-20 keV, collimator A. - (c) Energy ranges $0.6-1.2 \mathrm{keV}$ and $1.0-7.0 \mathrm{keV}$, collimator B.

From the altitude dependence of the counting rate in the lowest energy region, the emission of the atmospheric background was limited to altitudes below $180 \mathrm{~km}$. Thus only data free from the atmospheric background were used as cosmic X-rays.

Three kinds of counters gave consistent results in overlapping energy regions. The energy spectrum obtained with the xenon filled counters was slightly flatter than that with the argon filled counters. This seems to have been the case when Gorenstein et al. [1] observed Sco XR-1 on two different days, although they attributed the difference to the time variation of temperature. While we do not fully understand causes of the differences, we refer to results with the argon filled counters in the overlapping energy ranges, if any significant difference is found.

\section{Cyg XR-2}

Cyg XR-2 is significantly visible in the energy region above $0.6 \mathrm{keV}$, whereas it can not be seen in the lowest energy region. The energy spectrum of Cyg XR-2 is shown in Figure 4.

If this is represented by an empirical expression

$$
j(E)=E^{-1} \exp (-E / k T) \exp \left(-E_{a} / E\right),
$$

we obtain a dashed curve in Figure 4 for

$$
k T \simeq 4 \mathrm{keV}, \quad E_{a} \simeq 0.5 \mathrm{keV} .
$$


The value of $k T$ is consistent with other results, whereas the value of $E_{a}$ is much smaller than that given by Giacconi [2], $E_{a} \simeq 2 \mathrm{keV}$.

If the last factor in the expression (1) represents the absorption effect, it may be more realistic to use the expression,

$$
j(E)=E^{-1} \exp (-E / k T) \exp \left[-\left(E_{a} / E\right)^{b}\right]
$$

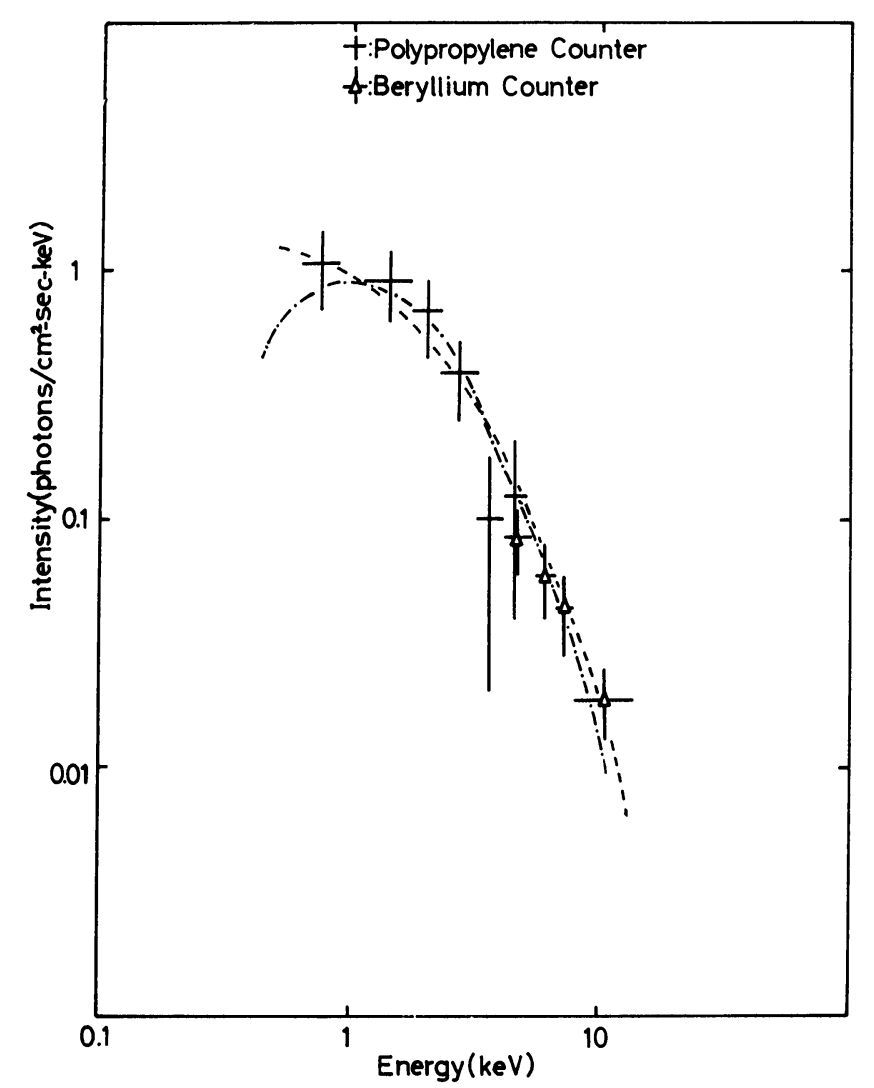

Fig. 4. The energy spectrum of Cyg XR-2. Calculated curves for Equation (1) with (2) and Equation (3) with (4) are shown by dashed and dot-dashed curves, respectively.

with $b \approx 8 / 3$. The spectrum shown by a dot-dashed curve in Figure 4 represents the expression (3) for

$$
k T=3.4 \mathrm{keV}, \quad E_{a}=0.8 \mathrm{keV} .
$$

The latter value of $E_{a}$ is related to the hydrogen column density by reference to the absorption cross section of interstellar gas of normal abundances [3],

$$
\int n_{\mathrm{H}} \mathrm{d} l \approx 3 \times 10^{21} \mathrm{~cm}^{-2}
$$


For the average hydrogen density of $\bar{n}_{\mathrm{H}}=0.8 \mathrm{~cm}^{-3}$ in the direction of Cyg XR-2, its distance is obtained as

$$
l \approx 1.2 \mathrm{kpc} \text {. }
$$

This is not inconsistent with the distance derived from the extinction of the possible optical counter part of Cyg XR-2.

\section{Diffuse Component}

The diffuse component of $\mathrm{X}$-rays with energies smaller than $1 \mathrm{keV}$ may show the effect of interstellar absorption. In order to see this effect, the intensities in the two lowest energy ranges are plotted against galactic latitude in Figure 5, in which the counts obtained with argon- and helium-filled counters with two different collimators are added up so as to increase statistics. The result is similar to the one given by Kraushaar [4].

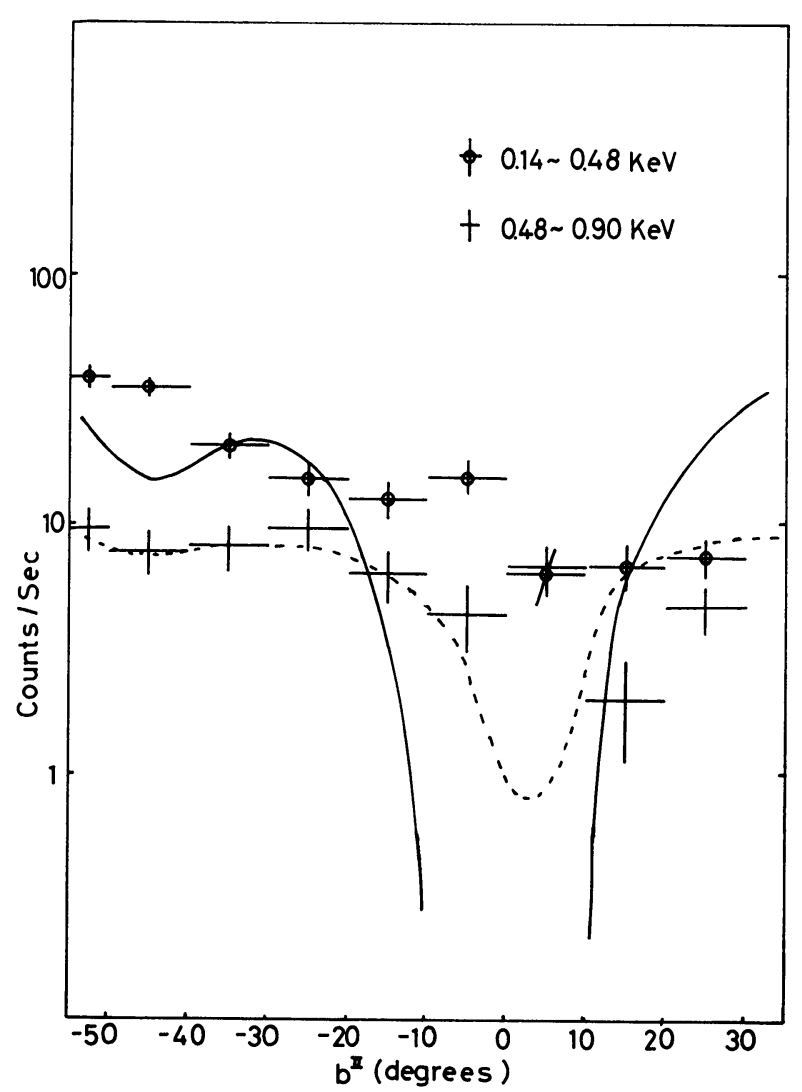

Fig. 5. The latitude dependence of counting rates in the energy ranges $0.14-0.48 \mathrm{keV}$ and 0.48 $0.9 \mathrm{keV}$. The solid $(0.14-0.48 \mathrm{keV})$ and dashed $(0.48-0.9 \mathrm{keV})$ curves represent the ones expected from interstellar absorption based on $21-\mathrm{cm}$ radio observation assuming zero helium abundance. The curves are normalized to the observed intensities at $-35^{\circ}$. 
From Figure 5 one sees that the latitude dependence is not symmetric about the galactic plane. This cannot be accounted for by the latitude dependence of the column density of neutral hydrogen as observed by $21-\mathrm{cm}$ radio emission. This is neither consistent with the one expected from the interstellar absorption based on $21-\mathrm{cm}$

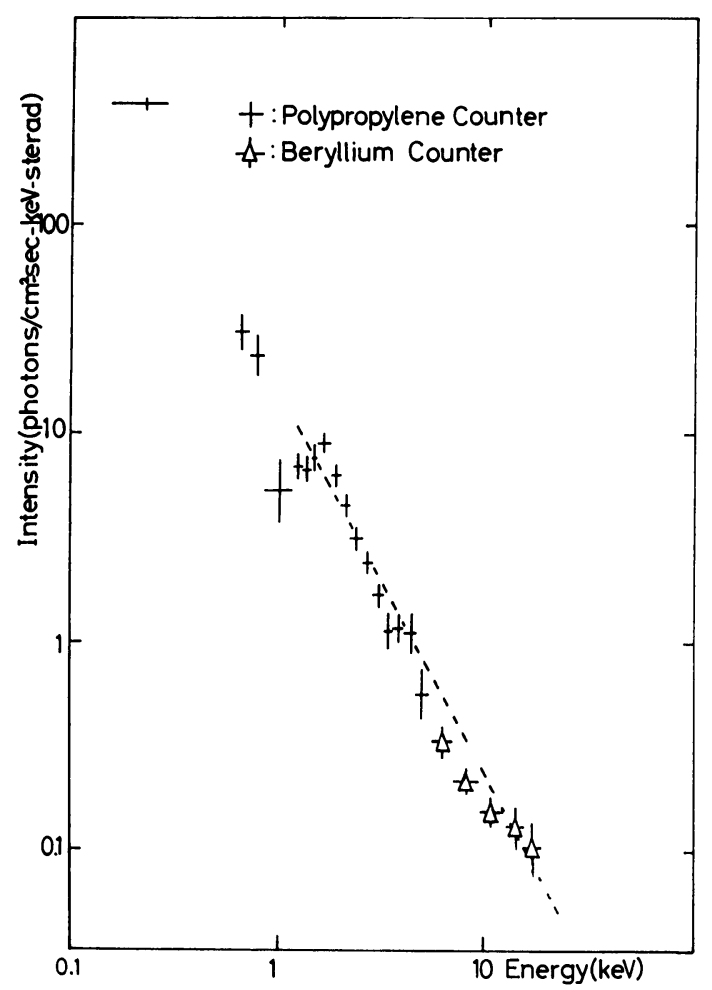

Fig. 6. The energy spectrum of the diffuse component at galactic latitudes between $-40^{\circ}$ and $-55^{\circ}$. The dashed line represents the $E^{-1.8}$ spectrum.

radio observation and the normal abundances of interstellar gas. Such a feature was also observed by Bowyer et al. [5]. This could be due, at least in part, to the emission of soft X-rays from unresolved sources in our galaxy.

Since effects of the interstellar medium are not yet known, the spectrum of background X-rays cannot be unambiguously derived. Here we give the spectrum at the highest latitude region scanned, namely between $b^{\mathrm{II}}=-40^{\circ}$ and $-55^{\circ}$. The spectrum shown in Figure 6 is approximately represented by a power law $E^{-1.8}$, except for a dip at about $1 \mathrm{keV}$. Although the reality of the dip should not be claimed too strongly at the present stage, this could again be due to a superposition of metagalactic X-rays subject to interstellar absorption and X-rays emitted in our galaxy. Except for the dip, the result is consistent with Kraushaar's. 


\section{References}

[1] Gorenstein, P., Gursky, H., and Garmire, G.: 1968, Astrophys. J. 153, 885.

[2] Giacconi, R.: this volume, p. 107.

[3] Bell, K. L. and Kingston, A. E.: 1967, Monthly Notices Roy. Astron. Soc. 136, 241.

[4] Bunner, A. N., Coleman, P. L., Kraushaar, W. L., McCammon, D., Palmieri, T. M., Shilepsky, A., and Ulmer, M.: 1969, Nature 223, 1222.

[5] Bowyer, C. S., Field, G. B., and Mack, J. E.: 1968, Nature 217, 32. 